\title{
Calendar of Events
}

\section{DOT 2009}

Deep Offshore Technology International Conference \& Exhibition

New Orleans, USA, 3-5 February 2009

http://dot09.events.pennnet.com/fl/index.cfm

\section{DINAME 2009}

$11^{\text {th }}$ International Symposium on Dynamic Problems of Mechanics

Angra dos Reis, RJ, Brazil, 2-6 March 2009

www.abcm.org.br/diname2009/

\section{MARSTRUCT 2009}

$2^{\text {nd }}$ International Conference on Marine Structures

Lisbon, Portugal, 16-18 March 2009

marstruct2009@mar.ist.utl.pt

\section{IWWWFB 2009}

$24^{\text {th }}$ International Workshop on Water and Floating Bodies Zelenogorsk, Russia, 19-22 April 2009

www.iwwwfb.org/

\section{OTC 2009}

$40^{\text {th }}$ Offshore Technology Conference

Houston, USA, 4-7 May 2009

www.otcnet.org/2009

\section{OMAE 2009}

$28^{\text {th }}$ International Conference on Ocean, Offshore and Arctic Engineering Honolulu, Hawaii, 31 May-5 June 2009

www.asmeconferences.org/omae2009

\section{ISOPE 2009}

$19^{\text {th }}$ International Offshore and Polar Engineering Conference Osaka, Japan, 21-26 June 2009

www.isope.org

\section{STAB 2009}

$10^{\text {th }}$ International Conference on Stability of Ships and Ocean Vehicles

St. Petersburg, Russia, 22-26 June 2009

www.stab2006.csa.ru

\section{ISSC 2009}

$17^{\text {th }}$ International Ship and Offshore Structures Congress

Seoul, Korea, 16-21 August 2009

www.issc.ac

\section{HYDROELASTICITY 2009}

$5^{\text {th }}$ International Conference on Hydroelasticity in Marine Technology

Southampton, UK, 8-10 September 2009

www.soton.ac.uk/ hyel09

\section{MCMC 2009}

$8^{\text {th }}$ IFAC Conference on Manoeuvring and Control of Marine Craft

Guarujá, SP, Brazil, 17-19 September 2009

http://www.mecanica-poliusp.org.br/07even/cont/mcmc/ home.html

\section{IMAM 2009}

$13^{\text {th }}$ International Congress of the International Maritime Association of the Mediterranean

Istanbul, Turkey, 12-15 October 2009

www.imam2009.itu.edu.tr

\section{COPINAVAL 2009}

XXI PanAmerican Conference of Naval Engineering, Maritime Transport and Port Engineering

Viña del Mar, Chile, 19-22 October 2009

www.copinaval.com

\section{COBEM 2009}

$20^{\text {th }}$ International Congress of Mechanical Engineering Gramado, RS, Brazil, 15-20 November 2009 www.abcm.org.br/cobem2009/

\section{PACAM 2010}

$11^{\text {th }}$ Pan-American Congress of Applied Mechanics Foz do Iguaçu, PR, Brazil, 4-8 January 2010 www.abcm.org.br/pacam2010/

\section{PRADS 2010}

$11^{\text {th }}$ International Symposium on Practical Design of Ships and Other Floating Structures

Rio de Janeiro, 19-24 September 2010

www.prads2010.org.br 